\title{
ON MODULES OVER MOTIVIC RING SPECTRA
}

\author{
ELDEN ELMANTO AND HÅKON KOLDERUP
}

\begin{abstract}
We provide an axiomatic framework that characterizes the stable $\infty$-categories that are module categories over a motivic spectrum. This is done by invoking Lurie's $\infty$-categorical version of the Barr-Beck theorem. As an application, this gives an alternative approach to Röndigs and Østvær's theorem relating Voevodsky's motives with modules over motivic cohomology and to Garkusha's extension of Röndigs and Østvær's result to general correspondence categories, including the category of Milnor-Witt correspondences in the sense of Calmès and Fasel. We also extend these comparison results to regular Noetherian schemes over a field (after inverting the residue characteristic), following the methods of Cisinski and Déglise.
\end{abstract}

\section{INTRODUCTION}

In [37] and [38], Röndigs and Østvær employed the technology of motivic functors developed in [18] to prove an important structural result regarding motivic cohomology, namely that there is an equivalence of model categories between motives and modules over motivic cohomology (at least over fields of characteristic zero). In particular, this implies that Voevodsky's triangulated categories of motives, introduced in [40], is equivalent to the homotopy category of modules over the motivic Eilenberg-Mac Lane spectrum. This result has been extended to bases which are regular schemes over a field in the work of Cisinski-Déglise on integral mixed motives in the equicharacteristic case [9]. More recently, Röndigs-Østvær's result was extended to general categories of correspondences over a field by Garkusha in [16]. These theorems provide pleasant reinterpretations of Voevodsky's category of motives as modules over a highly structured ring spectrum. The analog in topology is the result that chain complexes over a ring $R$ is equivalent (in an appropriate model categorical sense) to modules over the Eilenberg-Mac Lane spectrum HR. This result was first obtained by Schwede and Shipley in [39] as part of the characterization of stable model categories in loc. cit. ${ }^{1}$

In the present paper, we aim to provide a general axiomatic approach to the above results. More precisely, by making use of Lurie's $\infty$-categorical version of the Barr-Beck theorem we derive a characterization of those stable $\infty$-categories that are equivalent to a module category over a motivic spectrum. These categories are instances of motivic module categories as defined in Definition 3.1. Examples include $\operatorname{DM}(k)$ in the sense of Voevodsky [33] and $\widetilde{\mathrm{DM}}(k)$ in the sense of Déglise-Fasel [17]. Our characterization then reads as follows:

Theorem 1.1 (See Theorem 5.2). Let $k$ be a field of exponential characteristic e, and suppose that $\mathscr{M}(k)$ is a motivic module category on $k$. Then there is an equivalence of presentably symmetric monoidal stable $\infty$-categories

$$
\mathscr{M}(k)\left[\frac{1}{e}\right] \simeq \operatorname{Mod}_{R_{\mathscr{M}}\left[\frac{1}{e}\right]}(\mathrm{SH}(k)),
$$

where $R_{\mathscr{M}}$ is a motivic $\mathcal{E}_{\infty}$-ring spectrum in $\mathrm{SH}(k)$ corresponding to the monoidal unit in $\mathscr{M}(k)$. In particular, the associated triangulated categories are equivalent.

In fact, we formulate a parametrized version of motivic module categories and, under further hypotheses, we show that Theorem 1.1 extends to regular schemes over fields (see Theorem 5.5). The proof of the latter follows the approach of Cisinski-Déglise [9], while the proof of Theorem 1.1 breaks down into three steps:

(1) Invoke the Barr-Beck-Lurie theorem to prove that a motivic module category $\mathscr{M}(k)$ on $k$ is equivalent to the category of modules over some monad on $\mathrm{SH}(k)$.

(2) Produce a functor from modules over the monad to modules over a corresponding motivic spectrum (Lemma 3.6).

(3) Determine when this functor is an equivalence.

2010 Mathematics Subject Classification. 14F20, 14F42, 19E15, 55P42, 55 P43.

Key words and phrases. Motivic homotopy theory, motivic cohomology, Barr-Beck-Lurie, $\infty$-categories.

${ }^{1}$ We remark that an $\infty$-categorical treatement of the Schwede-Shipley results can be found in [30, Theorem 7.1.2.1]. 
After proving Theorem 1.1 we proceed to give a way to engineer several examples of motivic module categories via the notion of correspondence categories, on which one can apply the usual constructions of motivic homotopy theory.

1.1. Overview. Here is an outline of this paper:

- In Section 2 we collect some background material on the Barr-Beck-Lurie theorem, on compact rigid generation in motivic homotopy theory, and on premotivic categories.

- In Section 3 we provide an axiomatic framework characterizing the stable $\infty$-categories that are module categories over motivic spectra.

- In Section 4 we move on to discuss examples of categories satisfying the axioms of Section 3. The most prominent example are those arising from some sort of correspondences.

- Finally, in Section 5 we prove that the axioms of Section 3 are satisfied for the correspondence categories constructed in Section 4 in various situations.

1.2. Conventions and notation. We will rely on the language of $\infty$-categories following Lurie's books [29] and [30]. By a base scheme we mean a Noetherian scheme $S$ of finite dimension. We denote by Sch the category of Noetherian schemes, and by $\mathrm{Sm}_{S}$ the category of smooth schemes of finite type over $S$. The symbol $\mathbb{T}$ will denote the Thom space of the trivial vector bundle of rank 1 over the base $S$. Thus we have the standard motivic equivalences $\mathbb{T} \simeq \mathbf{A}^{1} / \mathbf{A}^{1} \backslash 0 \simeq \mathbb{P}^{1}$. We set $\mathbf{S}^{p, q}:=\left(S^{1}\right)^{\otimes(p-q)} \otimes \mathbf{G}_{m}^{\otimes q}$ and $\Sigma^{p, q} M:=\mathbf{S}^{p, q} \otimes M$, suitably interpreted in the category of motivic spaces or spectra. We reserve the symbol $\mathbf{1}$ for the motivic sphere spectrum in $\mathrm{SH}(k)$ and write $\Sigma^{p, q} \mathbf{1}$ for the $(p, q)$-suspension of $\mathbf{1}$. If $\tau$ is a topology on $\mathrm{Sm}_{S}$, we write $\mathrm{H}_{\tau}(S)$ (resp. $\mathrm{SH}_{\tau}(S)$ ) for the unstable (resp. the $\mathbb{T}$-stable) motivic homotopy $\infty$-category. If $\tau=$ Nis we may drop the decoration.

1.3. Acknowledgements. We would like to thank Paul Arne Østvær for suggesting to us the problem, and Shane Kelly for useful comments and suggestions. We would especially like to thank Tom Bachmann for very useful comments that changed the scope of this paper. Elmanto would like to thank John Francis for teaching him about "Barr-Beck thinking", Marc Hoyois for suggesting to him the alternative strategy to deriving [38] a long time ago, and Maria Yakerson for teaching him about MW-motives. Kolderup would like to thank Jean Fasel and Paul Arne Østvær for their patience and for always being available for questions.

\section{PRELIMINARIES}

2.1. The Barr-Beck-Lurie Theorem. Let us start out by recalling the Barr-Beck-Lurie theorem characterizing modules over a monad, in the setting of $\infty$-categories. We use the terminology of [26, §3.7].

Let $F: C \rightleftarrows \mathrm{D}: G$ be an adjunction. Then the endofunctor $G F: C \rightarrow C$ is a monad, and the functor $G: D \rightarrow C$ factors as

$$
\mathrm{D} \stackrel{\mathrm{G}^{\mathrm{enh}}}{\longrightarrow} \operatorname{LMod}_{G F}(\mathrm{C}) \stackrel{u}{\rightarrow} \mathrm{C},
$$

where $u$ is the forgetful functor. Moreover, the functor $G^{\text {enh }}: \mathrm{D} \rightarrow \operatorname{LMod}_{G F}(C)$ admits a left adjoint

$$
\mathrm{F}^{\mathrm{enh}}: \operatorname{LMod}_{G F}(\mathrm{C}) \rightarrow \mathrm{D} \text {. }
$$

2.1.1. The net result is that the adjunction $F: C \rightleftarrows D: G$ factors as

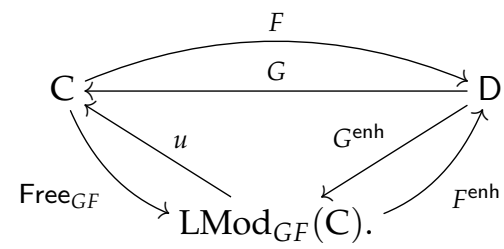

Here the functor Free $G F: C \rightarrow \operatorname{LMod}_{G F}(C)$ is simply the left adjoint to the functor $u$ appearing in the factorization of $G$ above, and thus deserves to be called the "free GF-module" functor. 
2.1.2. The Barr-Beck-Lurie theorem provides necessary and sufficient conditions for the functor $G^{\text {enh }}: D \rightarrow$ $\operatorname{LMod}_{G F}(\mathrm{C})$ to be an equivalence. Before stating the theorem, recall first that a simplicial object $X_{\bullet}: \Delta^{\text {op }} \rightarrow \mathrm{D}$ is split if it extends to a split augmented object; in other words it extends to a functor $U: \Delta_{-\infty}^{\mathrm{op}} \rightarrow \mathrm{D}$. Here $\Delta_{-\infty}$ is the category whose objects are integers $\geq-1$, and where $\operatorname{Hom}_{\Delta_{-\infty}}(n, m)$ consists of nondecreasing maps $n \cup\{-\infty\} \rightarrow m \cup\{-\infty\}$. Every split augmented simplicial diagram is a colimit diagram so that the map colim $X_{\bullet} \rightarrow X_{-1}$ is an equivalence. If $G: D \rightarrow C$ is a functor, we say that a simplicial object $X_{\bullet}$ in $D$ is $G$-split if $G \circ X_{\bullet}$ is split.

Theorem 2.1 (Barr-Beck-Lurie [30, Theorem 4.7.3.5]). Let $G: D \rightarrow C$ be a functor of $\infty$-categories admitting a left adjoint $F: \mathrm{C} \rightarrow \mathrm{D}$. Then the following are equivalent:

(1) The functor $\mathrm{G}^{\mathrm{enh}}$ and $\mathrm{F}^{\mathrm{enh}}$ are mutually inverse equivalences.

(2) The functor $\mathrm{G}^{\mathrm{enh}}$ is conservative, and for any simplicial object $X_{\bullet}: \Delta^{\mathrm{op}} \rightarrow \mathrm{D}$ which is G-split, $X_{\bullet}$ admits a colimit in $\mathrm{D}$. Furthermore, any extension $\overline{X_{\bullet}}:\left(\Delta^{\mathrm{op}}\right)^{\triangleright} \rightarrow \mathrm{D}$ is a colimit diagram if and only if $\mathrm{G} \circ \overline{X_{\bullet}}$ is.

Any adjunction $(F, G)$ satisfying the equivalent conditions above is called monadic.

2.2. Compact and rigid objects in motivic homotopy theory. We now recall some facts about compact-rigid generation in motivic stable $\infty$-categories.

2.2.1. For now we work over an arbitrary base $S$. Denote by:

(1) $\mathrm{SH}^{\omega}(S)$ the full subcategory of $\mathrm{SH}(S)$ spanned by the compact objects, and

(2) $\mathrm{SH}^{\mathrm{rig}}(S)$ the full subcategory of $\mathrm{SH}(S)$ spanned by the strongly dualizable objects.

The $\infty$-category $\mathrm{SH}(S)$ is generated under sifted colimits by $\Sigma_{\mathbb{T}}^{q} \Sigma_{\mathbb{T}}^{\infty} X_{+}$, where $X$ is an affine smooth scheme over $S$ and $q \in \mathbf{Z}$ [27, Proposition 4.2.4]. Furthermore, each generator is a compact object in $\mathrm{SH}(S)$ since Nisnevich sheafification preserves filtered colimits (see, for example, [22, Proposition 6.4] where we set the group of equivariance to be trivial). Hence the $\infty$-category $\mathrm{SH}^{\omega}(S)$ is generated under finite colimits and retracts by $\Sigma_{\mathbb{T}}^{q} \Sigma_{\mathbb{T}}^{\infty} X_{+}$, where $q \in \mathbf{Z}$ and $X$ is affine. In particular the unit in $\mathrm{SH}(S)$ is compact and we have an inclusion

$$
\mathrm{SH}^{\mathrm{rig}}(S) \subseteq \mathrm{SH}^{\omega}(S)
$$

Over fields this inclusion is an equality-at least after an appropriate localization:

Lemma 2.2. Let $k$ be a field and suppose that $\ell$ is a prime which is coprime to the exponential characteristic e of $k$. Let $L_{(\ell)}: \mathrm{SH}(k) \rightarrow \mathrm{SH}(k)$ be the localization endofunctor at $\ell$. Then (2.1) induces equalities

$$
\mathrm{SH}^{\mathrm{rig}}(k)_{(\ell)}=\mathrm{SH}^{\omega}(k)_{(\ell)}
$$

and

$$
\mathrm{SH}^{\mathrm{rig}}(k)\left[\frac{1}{e}\right]=\mathrm{SH}^{\omega}(k)\left[\frac{1}{e}\right] .
$$

Proof. Since $\mathrm{SH}^{\omega}(k)$ is generated as a stable subcategory which is closed under retracts by $\Sigma_{\mathbb{T}}^{\infty} X_{+}$, where $X$ is a smooth affine scheme, $\mathrm{SH}^{\omega}(k)_{(\ell)}$ is generated by the image of the same objects under $L_{(\ell)}$. Now, $\Sigma_{\mathbb{T}}^{\infty} X_{+}$is dualizable whenever $X$ is smooth and proper by [35], hence it suffices to prove that $L_{(\ell)}\left(\Sigma_{\mathbb{T}}^{\infty} X_{+}\right)$is a retract of some $L\left(\Sigma_{\mathbb{T}}^{\infty} Y_{+}\right)$, where $Y$ is a smooth projective $S$-scheme. If $k$ is perfect then this is [28, Corollary B.2]. We note that this result is extended to the case of arbitrary fields in [14, Theorem 3.2.1]. The result for $e$-inverted motivic spectra follows.

Example 2.3. If $S$ is a positive dimensional base scheme, we should not expect (2.1) to be an equality in general even after localization; see [8, Corollary 3.2.7].

2.2.2. We adopt the following terminology:

Definition 2.4. Let $k$ be a field and suppose that $L: \mathrm{SH}(k) \rightarrow \mathrm{SH}(k)$ is a localization endofunctor. We say that $L(\mathrm{SH}(k))$, or simply $L$, has compact-rigid generation if (2.1) is an equality after applying $L$.

Hence Lemma 2.2 tells us that $\mathrm{SH}(k)_{(\ell)}$ and $\mathrm{SH}(k)\left[\frac{1}{e}\right]$ have compact-rigid generation. 
2.3. Premotivic categories and adjunctions. Lastly, we recall Cisinski and Déglise's notion of a premotivic category [7]. Suppose that $\mathscr{S}$ is a full subcategory of the category Sch of Noetherian schemes, and let $\mathscr{P}$ denote a class of admissible morphisms [7, §1]. In fact, the only example we care about is when $\mathscr{P}$ is the class of smooth morphisms. As in [7, 11 (see also [10, Appendix A] for a more succint discussion), a functor

$$
\mathscr{M}: \mathscr{S}^{\mathrm{op}} \rightarrow \mathrm{Cat}_{\infty}
$$

is called a $\mathscr{P}$-premotivic category over $\mathscr{S}$ if for each morphism $f: T \rightarrow S$ in $\mathscr{S}$, the induced functor $f^{*}: \mathscr{M}(S) \rightarrow \mathscr{M}(T)$ admits a right adjoint $f_{*}$, and if $f$ is admissible, it admits a left adjoint $f_{\# \text {. The }}$ left adjoints are furthermore required to satisfy the $\mathscr{P}$-base change formula, i.e., the exchange morphism $E x_{\#}^{*}: q_{\# g^{*}} \rightarrow f^{*} p_{\#}$ is an equivalence whenever

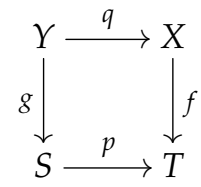

is a Cartesian diagram in $\mathscr{S}$ such that $p$ is a $\mathscr{P}$-morphism. See $[7, \S 1.1 .9]$ for details.

We refer the reader to the thesis of Khan [27] for a detailed discussion of premotivic categories in the $\infty$-categorical setting. If the context is clear, we simply refer to $\mathscr{M}$ as a premotivic category. We may also speak of premotivic categories taking values in other (large) $\infty$-categories such as $\mathrm{Cat}_{\infty}^{\otimes}, \mathrm{Cat}_{\infty, \text { stab }}$ or $\operatorname{Pr}^{L}$.

2.3.1. We also have the appropriate notion of an adjunction between premotivic categories (see [7, Definition 1.4.6], [10, Definition A.1.7]). Indeed, if $\mathscr{M}$ and $\mathscr{M}^{\prime}$ are premotivic categories, then a premotivic adjunction is a transformation $\gamma^{*}: \mathscr{M} \rightarrow \mathscr{M}^{\prime}$ such that

(1) for each $S \in \mathscr{S}$, the functor $\gamma_{S}^{*}: \mathscr{M}(S) \rightarrow \mathscr{M}^{\prime}(S)$ admits a right adjoint $\gamma_{S *}$.

(2) For each morphism $f: T \rightarrow S \in \mathscr{S}$, the canonical transformation $f_{\#} \gamma_{S}^{*} \rightarrow \gamma_{T}^{*} f_{\#}$ is an equivalence.

Furthermore we say that a premotivic adjunction $\gamma^{*}$ is a localization of premotivic categories (or, simply, a localization) if for each $S \in \mathscr{S}$ the functor $\gamma_{S *}$ is fully faithful, i.e., a localization in the sense of [29, Definition 5.2.7.2]. We say that a localization of premotivic categories is smashing if $\gamma_{S *}$ preserves colimits. Suppose further that $\mathscr{M}$ takes values in $\mathrm{Cat}_{\infty}^{\otimes}$. In particular, the functors $f^{*}$ are strongly symmetric monoidal. Then a localization $L$ is symmetric monoidal if given any $S \in \mathscr{S}$ and any $\mathrm{E} \in \mathscr{M}(S)$ that is $L$-local, then for any $\mathrm{F} \in \mathscr{M}(S), \mathrm{E} \otimes \mathrm{F}$ is $L$-local as well. This last condition implies that the symmetric monoidal structure on $\mathscr{M}(S)$ descends to one on the subcategory of $L$-local objects and that the localization functor is strongly symmetric monoidal [30, Proposition 2.2.1.9].

2.3.2. We recall two conditions on $\mathscr{M}$ which will be relevant to us later. In order to formulate them, we will now assume that $\mathscr{M}$ takes values in stable $\infty$-categories. Let $S \in \mathscr{S}$ be a scheme. Suppose that $i: Z \rightarrow S$ is a closed subscheme, and let $j: U \rightarrow S$ be its open complement.

Definition 2.5. Let $\mathscr{M}: \mathscr{S}^{\mathrm{op}} \rightarrow$ Cat $_{\infty, \text { stab }}$ be a premotivic category, and let $Z \stackrel{i}{\rightarrow} S \stackrel{j}{\leftarrow} U$ be as above. We say that $\mathscr{M}$ satisfies $\left(\operatorname{Loc}_{i}\right)$ if

$$
\mathscr{M}(Z) \stackrel{i_{*}}{\rightarrow} \mathscr{M}(S) \stackrel{j^{*}}{\rightarrow} \mathscr{M}(U)
$$

is a cofiber sequence of stable $\infty$-categories. We say that $\mathscr{M}$ satisfies (Loc) if $\left(\operatorname{Loc}_{i}\right)$ is satisfied for any closed immersion $i$.

Now let $c=\left(c_{\alpha}\right)_{\alpha \in I}$ be a collection of Cartesian sections of $\mathscr{M}$ (the only case we consider is $\left\{\Sigma^{p, q} \mathbf{1}\right\}_{p, q \in \mathbf{Z}}$ ). We denote by $\mathscr{M}_{\mathcal{C}}(S) \subseteq \mathscr{M}(S)$ the smallest thick subcategory of $\mathscr{M}(S)$ which contains $f_{\#} f^{*} c_{\alpha, X}$ for any smooth morphism $f: T \rightarrow S$. Following [9, Definition 2.3], we call objects in $\mathscr{M}_{c}(S)$ c-constructible. We say that $\mathscr{M}$ is $c$-generated if for all $X \in \mathscr{S}$ the stable $\infty$-category $\mathscr{M}(S)$ is generated by $\mathscr{M}_{c}(S)$ under all small colimits.

Definition 2.6. Let $\mathscr{M}: \mathscr{S}^{\mathrm{op}} \rightarrow \mathrm{Cat}_{\infty, \text { stab }}$ be a premotivic category. Suppose that $\mathscr{A} \subseteq \mathscr{S}^{\Delta^{1}}$ is a collection of morphisms in $\mathscr{S}$. We say that $\mathscr{M}$ is continuous with respect to $\mathscr{A}$ if the following holds. Suppose that $X: I \rightarrow \mathscr{S}$ is a cofiltered diagram in $\mathscr{S}$ whose transition maps belongs to $\mathscr{A}$ and whose limit $X:=\lim _{\alpha \in I} X_{\alpha}$ exists in $\mathscr{S}$. Then the canonical map

$$
\mathscr{M}_{c}(X) \rightarrow \lim _{\alpha \in I} \mathscr{M}_{c}\left(X_{\alpha}\right) .
$$


is an equivalence.

\section{Motivic MOdule CATEGORIES}

In this section we formulate the notion of motivic module categories and relate it to categories of modules over a motivic $\mathcal{E}_{\infty}$-ring spectrum.

3.0.1. Let $\mathscr{S}$ be a full subcategory of Sch. By $[2,7]$ we then have a premotivic category $\left.\mathrm{SH}\right|_{\mathscr{S}}: \mathscr{S} \rightarrow \operatorname{Pr}_{\text {stab }}^{L, \otimes}$ whose value at $S \in \mathscr{S}$ is the motivic stable homotopy category $\mathrm{SH}(S)$ over $S$.

Definition 3.1. Let $\mathscr{S}$ be as above, and suppose that $L:\left.\left.\mathrm{SH}\right|_{\mathscr{S}} \rightarrow L(\mathrm{SH})\right|_{\mathscr{S}}$ is a localization which is symmetric monoidal in the sense of $\S 2.3 .1$. We then define the following:

(1) Let $S \in \mathscr{S}$. An L-local motivic module category on $S$ is a presentably symmetric monoidal stable $\infty$-category $\mathscr{M}(S)$ equipped with an adjunction

$$
\gamma_{S}^{*}: L(\mathrm{SH}(S)) \rightleftarrows \mathscr{M}(S): \gamma_{S *}
$$

such that the left adjoint $\gamma_{S}^{*}$ is symmetric monoidal, and the right adjoint $\gamma_{S *}$ is conservative and preserves sifted colimits.

(2) An L-local motivic module category over $\mathscr{S}$ (or, simply, a motivic module category if the context is clear) is a premotivic category

$$
\mathscr{M}: \mathscr{S}^{\mathrm{op}} \rightarrow \operatorname{Pr}_{\text {stab }}^{L, \otimes}
$$

valued in presentably symmetric monoidal stable $\infty$-categories, along with a premotivic adjunction

$$
\gamma^{*}:\left.L(\mathrm{SH})\right|_{\mathscr{S}} \rightarrow \mathscr{M} ; \quad S \mapsto\left(\gamma_{S}^{*}: L(\mathrm{SH}(S)) \rightarrow \mathscr{M}(S)\right),
$$

which evaluates to an $L$-local motivic module category $\mathscr{M}(S)$ on $S$ for each $S \in \mathscr{S}$.

If $L$ is the identity functor, then we simply say that $\mathscr{M}$ is a motivic module category. When the localization $L$ is clear, we may denote a motivic module category by a pair $\left(\left.\mathrm{SH}\right|_{\mathscr{S}, \mathscr{M}}\right)$. Moreover, if the scheme $S$ is implicitly understood, we may drop the $S$ from the notation $\left(\gamma_{S}^{*}, \gamma_{S *}\right)$.

In $\S 4$ we will give a way to construct motivic module categories using very general inputs.

Lemma 3.2. Let $S \in \mathscr{S}$, and let $\mathbf{1}_{S} \in \mathrm{SH}(S)$ denote the motivic sphere spectrum over $S$. If $\mathscr{M}$ is an L-local motivic module category, then the spectrum $L \gamma_{*} \gamma^{*}\left(\mathbf{1}_{S}\right) \in \mathrm{SH}(S)$ is an $\mathcal{E}_{\infty}$-ring spectrum.

Proof. As $\gamma_{*}$ is lax symmetric monoidal, it follows that $\gamma_{*}$ preserves $\mathcal{E}_{\infty}$-algebras. Since $\gamma^{*}$ is symmetric monoidal, $\gamma^{*}\left(\mathbf{1}_{S}\right)$ is the unit object in $\mathscr{M}$ and is thus an $\mathcal{E}_{\infty}$-algebra. As $L$ is symmetric monoidal, we conclude that $\gamma_{*} \gamma^{*}\left(\mathbf{1}_{S}\right)$ is an $\mathcal{E}_{\infty}$-ring spectrum.

3.0.2. The Barr-Beck-Lurie theorem ensures that a motivic module category on $S$ is always equivalent to modules over a monad, as the following lemma records. We will subsequently investigate when we can further enhance this equivalence to modules over the $\mathcal{E}_{\infty}$-ring spectrum $L \gamma_{*} \gamma^{*}\left(\mathbf{1}_{S}\right)$.

Lemma 3.3. If $\mathscr{M}(S)$ is a motivic module category on $S$, then the induced adjunction

$$
\gamma^{*, \text { enh }}: \operatorname{LMod}_{\gamma_{*} \gamma^{*}}(L(\mathrm{SH}(S))) \rightleftarrows \mathscr{M}(S): \gamma_{*}^{\text {enh }}
$$

is an equivalence of $\infty$-categories.

Proof. By assumption, the conditions of Theorem 2.1 are satisfied.

3.1. Motivic module categories versus categories of modules. The following definition will be essential in relating a motivic module category to a category of modules over a motivic $\mathcal{E}_{\infty}$-ring spectrum.

Definition 3.4. Let $\mathscr{M}$ be an $L$-local motivic module category over $\mathscr{S}$ and let $S \in \mathscr{S}$. We say that the pair $\left(\left.\mathrm{SH}\right|_{\mathscr{S}}, \mathscr{M}\right)$ admits the projection formula at $S$ if there is an equivalence

$$
\gamma_{*} \gamma^{*}\left(\mathbf{1}_{S}\right) \otimes(-) \stackrel{\simeq}{\rightarrow} \gamma_{*} \gamma^{*}
$$

of endofunctors on $L(\mathrm{SH}(S))$. If $(\mathrm{SH} \mid \mathscr{S}, \mathscr{M})$ admits the projection formula at any $S \in \mathscr{S}$, we say that $\left(\left.\mathrm{SH}\right|_{\mathscr{S}, \mathscr{M}}\right)$ admits the projection formula. 
Theorem 3.5. Let $\mathscr{M}$ be an L-local motivic module category over $\mathscr{S}$. Suppose that $S \in \mathscr{S}$ is a scheme such that $\left(\left.\mathrm{SH}\right|_{\mathscr{S}}, \mathscr{M}\right)$ admits the projection formula at $\mathrm{S}$. Then there is an equivalence of presentably symmetric monoidal stable $\infty$-categories

$$
\mathscr{M}(S) \simeq \operatorname{Mod}_{L \gamma_{*} \gamma^{*}\left(\mathbf{1}_{S}\right)}(\mathrm{SH}(S)) .
$$

Consequently, if $\left(\left.\mathrm{SH}\right|_{\mathscr{S}}, \mathscr{M}\right)$ admits the projection formula, then we have an equivalence of premotivic categories

$$
\mathscr{M} \simeq \operatorname{Mod}_{L \gamma_{*} \gamma^{*}(\mathbf{1})}(\mathrm{SH}(-)) \text {. }
$$

3.1.1. In light of Lemma 3.3, we can prove Theorem 3.5 by means of relating modules over the monad $\gamma_{*} \gamma^{*}$ with modules over the motivic spectrum $\gamma_{*} \gamma^{*}\left(\mathbf{1}_{S}\right)$. Thus, given $S \in \mathscr{S}$ our task is to formulate a relationship between the two $\infty$-categories

$$
\operatorname{LMod}_{\gamma_{*} \gamma^{*}}(\mathrm{SH}(S)) \text { and } \operatorname{LMod}_{\gamma_{*} \gamma^{*}\left(\mathbf{1}_{S}\right) \otimes(-)}(\mathrm{SH}(S)) .
$$

To do so, it suffices produce a map of monads

$$
c: \gamma_{*} \gamma^{*}\left(\mathbf{1}_{S}\right) \otimes(-) \rightarrow \gamma_{*} \gamma^{*},
$$

which will induce a functor

$$
c^{*}: \operatorname{LMod}_{\gamma_{*} \gamma^{*}\left(\mathbf{1}_{S}\right) \otimes(-)}(\mathrm{SH}(S)) \rightarrow \operatorname{LMod}_{\gamma_{*} \gamma^{*}}(\mathrm{SH}(S)) .
$$

For this, we appeal to a general lemma.

Lemma 3.6. Let $\mathrm{C}, \mathrm{D}$ be symmetric monoidal $\infty$-categories and suppose that we have an adjunction $F: \mathrm{C} \rightleftarrows \mathrm{D}: G$ such that $F$ is symmetric monoidal (so that $G$ is lax symmetric monoidal). Then there is a map of monads

$$
c: G F(1) \otimes(-) \rightarrow G F,
$$

which gives rise to a commutative diagram of adjunctions

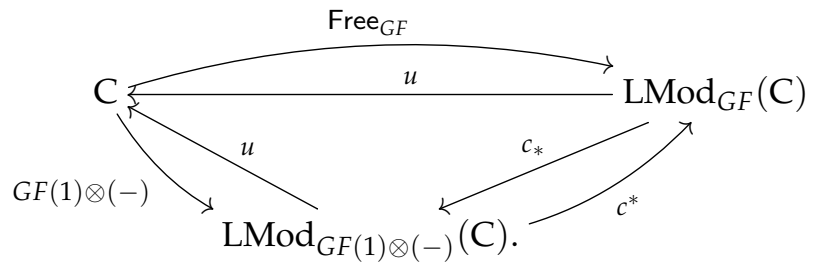

Proof. Since $F$ is monoidal and $G$ is lax monoidal, the functor $G F$ is lax monoidal. Hence $G F(1)$ is an algebra object of $C$, and thus $G F(1) \otimes(-)$ is indeed a monad. We construct the map of monads $c: G F(1) \otimes(-) \rightarrow$ $G F(-)$ by letting $c$ be the composite of the following maps of monads:

$$
\begin{aligned}
G F(1) \otimes(-) & \simeq(G F(1) \otimes(-)) \circ \mathrm{id} \\
& \stackrel{\mathrm{id} \circ \epsilon}{\longrightarrow}(G F(1) \otimes(-)) \circ G F(-) \\
& \stackrel{\mu}{\rightarrow} G(F(1) \otimes F(-)) \\
& \simeq G F .
\end{aligned}
$$

Here $\epsilon$ is the unit of the adjunction $(F, G)$. The transformation $\epsilon$ is a map of monads via the triangle identities, and the map id $\circ \epsilon$ is a map of monads since we are o-tensoring two maps of monads. The map $\mu$ is given by the lax monoidal structure of $G$; more precisely, we note that the endofunctor $G(A \otimes F(-))$ is a monad for any algebra object $A$, and so $G(F(1) \otimes F(-))$ is in particular a monad. We have a canonical equivalence of monads

$$
(G F(1) \otimes(-)) \circ G F(-) \simeq G F(1) \otimes G F(-) .
$$

The lax structure of $G$ then provides a morphism of endofunctors

$$
G F(1) \otimes G F(-) \rightarrow G(F(1) \otimes F(-)) \simeq G F(-),
$$

and the lax structure also verifies that this is a map of monads. This gives rise to a functor $\mathcal{C}_{*}: \operatorname{LMod}_{G F}(\mathrm{C}) \rightarrow$ $\operatorname{LMod}_{G F(1) \otimes(-)}(C)$, which has a left adjoint by the adjoint functor theorem. 
To obtain the desired factorizations, we note that we have the following commutative diagram of forgetful functors

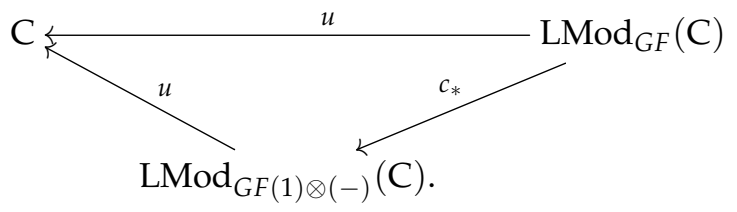

Thus the left adjoints also commute.

\subsubsection{We can now apply Lemma 3.6 to prove Theorem 3.5.}

Proof of Theorem 3.5. We claim that the adjunction of Lemma 3.6,

$$
c^{*}: \operatorname{LMod}_{\gamma_{*} \gamma^{*}\left(\mathbf{1}_{S}\right)}(\mathrm{SH}(S)) \rightleftarrows \operatorname{LMod}_{\gamma_{*} \gamma^{*}}(\mathrm{SH}(S)): c_{*},
$$

is an equivalence. By the construction in the proof of Lemma 3.6, the above adjunction arises from a map of monads given by $c: \gamma_{*} \gamma^{*}\left(\mathbf{1}_{S}\right) \otimes(-) \rightarrow \gamma_{*} \gamma^{*}$. Since $(\mathrm{SH} \mid \mathscr{S}, \mathscr{M})$ satisfies the projection formula, we conclude that the adjunction $\left(c^{*}, c_{*}\right)$ is an equivalence.

Now, note that Theorem 2.1 and Lemma 3.6 are phrased for $\mathcal{E}_{1}$-algebras and left modules. However, as $\gamma_{*} \gamma^{*}(\mathbf{1})$ is an $\mathcal{E}_{\infty}$-ring spectrum by Lemma 3.2, the $\infty$-categories of left and right $\gamma_{*} \gamma^{*}(\mathbf{1})$-modules are equivalent. We thus conclude that there is an natural equivalence

$$
\operatorname{Mod}_{\gamma_{*} \gamma^{*}\left(\mathbf{1}_{S}\right)}(\mathrm{SH}(S)) \simeq \mathscr{M}(S)
$$

of $\infty$-categories, which carries $\gamma_{*} \gamma^{*}\left(\mathbf{1}_{S}\right)$ to the unit object $\gamma^{*}\left(\mathbf{1}_{S}\right)$ of $\mathscr{M}(S)$. Finally, if $\mathscr{M}$ satisfies the projection formula at any $S \in \mathscr{S}$, then the naturality of the above equivalence furnishes the equivalence of premotivic categories $\mathscr{M} \simeq \operatorname{Mod}_{\gamma_{*} \gamma^{*}(\mathbf{1})}(\mathrm{SH}(-))$.

Remark 3.7. In fact, the above reduction can be achieved using a more refined version of Lurie's Barr-Beck theorem [30, Proposition 4.8.5.8].

Remark 3.8. We were also informed by Niko Naumann that the above result is a consequence of [32, Proposition 5.29].

In the following Sections 4 and 5 we will provide examples for which the hypotheses of Theorem 3.5 are satisfied.

\section{Correspondence CATEgories}

The prime examples of motivic module categories are built from various notions of correspondences. In this section we will give an axiomatization of $\infty$-categories that behave like the category of framed correspondences as in [12]; Suslin-Voevodsky's category of finite correspondences [42], [33, Chapters 1 and 2]; Calmès and Fasel's finite Milnor-Witt correpondences [6,17]; Grothendieck-Witt correspondences [15]; and, more recently, the categories of correspondences studied in [11] and [13]. These examples will be discussed in $\S 4.2$. To begin with, consider the discrete category $\mathrm{Sch}_{S+}$, whose objects are $S$-schemes of the form $X_{+}:=X \amalg S$ and morphisms which preserve the base point. We consider the subcategory $\mathrm{Sm}_{S+} \subseteq \mathrm{Sch}_{S+}$ spanned by smooth $S$-schemes of finite type. We will use heavily the nonabelian derived $\infty$-category $\mathrm{P}_{\Sigma}(\mathrm{C})$ associated to an $\infty$-category $\mathrm{C}$ with finite products; more detailed treatments of this construction can be found in [5, Chapter 1] and [29, §5.5.8].

Definition 4.1. A correspondence category (over a base scheme $S$ ) is a preadditive ${ }^{2} \infty$-category C equipped with a graph functor

$$
\gamma_{C}: \mathrm{Sm}_{S+} \rightarrow \mathrm{C}
$$

satisfying the following conditions:

\footnotetext{
${ }^{2}$ Recall that a preadditive $\infty$-category is one that is pointed, has finite products and coproducts, and is such that the map $X \amalg Y \rightarrow$ $X \times Y$ is an equivalence for all $X, Y \in C$.
} 
(1) The functor $\gamma_{C}$ is essentially surjective and preserves finite coproducts, ${ }^{3}$ so that we get an induced functor

(2) The composite functor

$$
\gamma_{*}: \mathrm{P}_{\Sigma}(\mathrm{C}) \rightarrow \mathrm{P}\left(\mathrm{Sm}_{S}\right) ; \quad \mathscr{F} \mapsto \mathscr{F} \circ \gamma_{C}
$$

$$
\mathrm{Sm}_{S_{+}} \rightarrow \mathrm{C} \rightarrow \mathrm{P}_{\Sigma}(\mathrm{C}) \stackrel{\gamma_{*}}{\rightarrow} \mathrm{P}_{\Sigma}\left(\mathrm{Sm}_{S_{+}}\right)
$$

has a right lax $\mathrm{Sm}_{S+}$-linear structure. We abusively denote the composite (4.2) by $\gamma_{C}(-)$; the context will always make it clear what is meant.

The $\infty$-category CorrCat of correspondence categories is defined as a full subcategory of the (large) $\infty$ category PreAdd $\omega_{\infty, \mathrm{Sm}_{S+}}$ / of small preadditive $\infty$-categories and functors that preserve finite coproducts equipped with a finite coproduct-preserving functor from $\mathrm{Sm}_{S_{+}} \cdot{ }^{4}$

4.0.1. We begin with a couple of clarifying remarks and an example.

Remark 4.2. Informally, the $\mathrm{Sm}_{S+}$-linear structure on $\gamma_{C}(-)$ encodes, for any $X, Y \in \mathrm{Sm}_{S}$, maps

$$
X_{+} \otimes \gamma_{C}\left(Y_{+}\right) \rightarrow \gamma_{C}\left(X_{+} \otimes Y_{+}\right)
$$

in $\mathrm{P}_{\Sigma}\left(\mathrm{Sm}_{S+}\right) \simeq \mathrm{P}_{\Sigma}\left(\mathrm{Sm}_{S}\right)_{*}$ which are subject to various compatibilites. For example, if $f: X_{+} \rightarrow Z_{+}$is a map in $\mathrm{Sm}_{S+}$ then we have a 2-cell witnessing the commutativity of

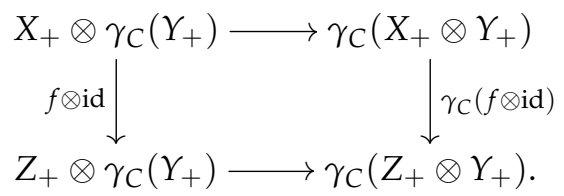

Similarly, if $g: Y_{+} \rightarrow Z_{+}$is a map in $\mathrm{Sm}_{S_{+}}$then we have a 2-cell witnessing the commutativity of

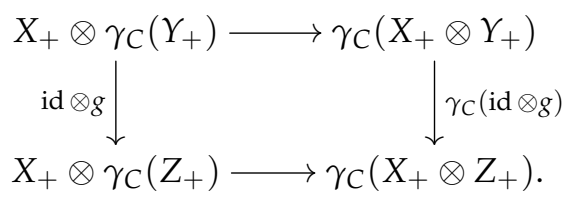

These cells are required to satisfy an infinite list of coherences.

Remark 4.3. The $\mathrm{Sm}_{S_{+}}$-linearity assumption will be satisfied if $\mathrm{C}$ has a symmetric monoidal structure and the functor $\gamma_{C}$ is symmetric monoidal. In more detail, we denote by CorrCat ${ }^{\otimes}$ the $\infty$-category of preadditive $\infty$-categories with a symmetric monoidal structure such that the graph functor $\gamma_{C}: S m_{S+} \rightarrow C$ is symmetric monoidal, essentially surjective and preserves finite coproducts. There is a forgetful functor CorrCat $^{\otimes} \rightarrow$ CorrCat; the second part of Definition 4.1 is obtained from the strong symmetric monoidality of $\gamma_{C}$. This is the case in the examples considered in this paper, but we include it as an axiom to clarify proofs of certain properties.

Example 4.4. Let Corr $S$ denote the discrete category whose objects are smooth $S$-schemes and morphisms are spans $X \leftarrow Y \rightarrow Z$. This is a preadditive category by [5, Lemma C.3]. The graph functor witnesses Corr $S_{S}$ as a correspondence category.

4.0.2. We now provide some elementary properties of a correspondence category.

Proposition 4.5. Let $\mathrm{C}$ be a preadditive $\infty$-category equipped with an essential surjection

$$
\gamma_{C}: \mathrm{Sm}_{k} \rightarrow \mathrm{C}
$$

which preserves coproducts, and let $\gamma_{\mathrm{C} *}$ denote the induced functor

$$
\gamma_{C *}: \mathrm{P}_{\Sigma}(\mathrm{C}) \rightarrow \mathrm{P}_{\Sigma}\left(\mathrm{Sm}_{S}\right) ; \quad \mathscr{F} \mapsto \mathscr{F} \circ \gamma_{C}
$$

Then the following properties hold:

\footnotetext{
${ }^{3}$ By requiring the functor $\gamma_{C}$ to preserve finite coproducts we include also the empty coproduct, ensuring that $\gamma_{C}$ preserves the base point of $\mathrm{Sm}_{S_{+}}$.

${ }^{4}$ More succinctly, CorrCat is the pullback of $\infty$-categories PreAdd $\times_{\text {Cat }{ }_{\infty}^{\amalg}}\left\{\mathrm{Sm}_{S+}\right\}$, where Cat ${ }^{\amalg}$ denotes $\infty$-categories with finite coproducts and finite coproduct-preserving functors.
} 
(1) The $\infty$-category $\mathrm{P}_{\Sigma}(\mathrm{C})$ is presentable and preadditive.

(2) The functor $\gamma_{C *}$ preserves sifted colimits.

(3) The functor $\gamma_{C *}$ is conservative.

Proof. Presentability of $\mathrm{P}_{\Sigma}(\mathrm{C})$ is [29, Proposition 5.5.8.10 (1)], while $\mathrm{P}_{\Sigma}$ applied to a preadditive $\infty$-category is again preadditive by [21, Corollary 2.4]. The functor $\gamma_{C *}$ preserves sifted colimits since sifted colimits are computed pointwise (a direct consequence of parts (4) and (5) of [29, Proposition 5.5.8.10]), while $\gamma_{C *}$ is conservative since $\gamma_{C}$ is essentially surjective.

4.0.3. The composite of $\gamma_{C}$ with Yoneda functor $\mathrm{Sm}_{S+} \stackrel{\gamma_{C}}{\rightarrow} \mathrm{C} \stackrel{y}{\rightarrow} \mathrm{P}_{\Sigma}(\mathrm{C})$ has a canonical sifted colimitpreserving extension $\gamma_{C}^{*}: P_{\Sigma}\left(S_{S+}\right) \rightarrow P_{\Sigma}(C)$. It is easy to check that $\gamma_{C *}$ is the right adjoint to $\gamma_{C}^{*}$ and thus $\gamma_{C}^{*}$ preserves all small colimits. As a result, we have an adjunction

$$
\gamma_{C}^{*}: \mathrm{P}_{\Sigma}\left(\mathrm{Sm}_{S+}\right) \rightleftarrows \mathrm{P}_{\Sigma}(\mathrm{C}): \gamma_{C *} .
$$

It is also easy promote the $S m_{S+}$-linear structure given by the second axiom of a correspondence category to a $\mathrm{P}_{\Sigma}\left(\mathrm{Sm}_{S_{+}}\right)$-linear structure so that the functor

extends to a right lax $\mathrm{P}_{\Sigma}\left(\mathrm{Sm}_{S+}\right)$-linear functor.

$$
\gamma_{C *} \circ \gamma_{C}^{*}: \mathrm{P}_{\Sigma}\left(\mathrm{Sm}_{S+}\right) \rightarrow \mathrm{P}_{\Sigma}\left(\mathrm{Sm}_{S_{+}}\right)
$$

4.0.4. Now we would like to do motivic homotopy theory on C. Recall that if $X, Y \in \mathrm{P}_{\Sigma}\left(\mathrm{Sm}_{S_{+}}\right)$, then $X$ is $\mathbf{A}^{1}$-homotopy equivalent to $Y$ if there are maps $f: X \rightarrow Y, g: Y \rightarrow X$ and $\mathbf{A}^{1}$-homotopies $H: \mathbf{A}_{+}^{1} \otimes X \rightarrow X$, $H^{\prime}: \mathbf{A}_{+}^{1} \otimes Y \rightarrow Y$ from $g f$ and $f g$ to the respective identity morphisms. We note that any $\mathbf{A}^{1}$-homotopy equivalence is an $\mathrm{L}_{\mathbf{A}^{1}}$-equivalence [34, $§ 2$ Lemma 3.6].

Lemma 4.6. The functor $\gamma_{C}: \mathrm{P}_{\Sigma}\left(\mathrm{Sm}_{S+}\right) \rightarrow \mathrm{P}_{\Sigma}\left(\mathrm{Sm}_{S+}\right)$ preserves $\mathbf{A}^{1}$-homotopy equivalences.

Proof. Suppose that we have a homotopy $H: \mathbf{A}_{+}^{1} \otimes X_{+} \rightarrow Y$ between maps $f, g: X \rightarrow Y$. We obtain, using the right lax-structure, a homotopy

$$
\mathbf{A}_{+}^{1} \otimes \gamma_{C}(X) \rightarrow \gamma_{C}\left(\mathbf{A}^{1} \times X\right) \rightarrow \gamma_{C}(Y)
$$

between $\gamma_{C}(f)$ and $\gamma_{C}(g)$.

Lemma 4.7. The functor $\gamma_{C}: \mathrm{P}_{\Sigma}\left(\mathrm{Sm}_{S+}\right) \rightarrow \mathrm{P}_{\Sigma}\left(\mathrm{Sm}_{S+}\right)$ preserves $\mathrm{L}_{\mathbf{A}^{1-e q u i v a l e n c e s .}}$

Proof. By definition the class of $\mathrm{L}_{\mathbf{A}^{1}}$-equivalences is the strong saturation, in the sense of [29, Proposition 5.5.4.5], of the maps in $P_{\Sigma}\left(S_{S}\right)$ by the (Yoneda image of) $\mathbf{A}^{1}$-projections $\pi_{X}:\left(\mathbf{A}^{1} \times X\right)_{+} \simeq \mathbf{A}_{+}^{1} \otimes X_{+} \rightarrow X_{+}$ for $X \in \mathrm{Sm}_{S}$. According to [5, Lemma 2.10] the class of $\mathrm{L}_{\mathbf{A}^{1}}$-equivalences is then generated under 2-out-of-3 and sifted colimits by maps of the form $\pi_{X} \amalg \mathrm{id}_{Y_{+}}$where $Y \in \mathrm{Sm}_{S}$.

Since $\pi_{X}$ is an $\mathbf{A}^{1}$-homotopy equivalence, it follows from Lemma 4.6 that $\gamma_{C}\left(\pi_{X}\right)$ is an $\mathbf{A}^{1}$-homotopy equivalence. Since $\gamma_{C}$ preserves coproducts by assumption, the same is true for the morphism

$$
\gamma_{C}\left(\pi_{X} \amalg \operatorname{id}_{Y_{+}}\right) \simeq \gamma_{C}\left(\pi_{X}\right) \amalg \gamma_{C}\left(\operatorname{id}_{Y_{+}}\right) .
$$

The functor $\gamma_{C}$ clearly preserves the 2-out-of-3-property. Lastly, the functor $\gamma_{C}$ preserves sifted colimits by definition and sifted colimits are computed valuewise in $\mathrm{P}_{\Sigma}\left(\mathrm{Sm}_{S+}\right)^{\Delta^{1}}$. Hence we conclude that $\gamma_{C}$ preserves $\mathrm{L}_{\mathbf{A}^{1}}$-equivalences.

4.0.5. Now we take into account a topology that we might want to put on $\mathrm{Sm}_{S_{+}}$, namely, the topology of coproduct decomposition. This is a topology on $\mathrm{Sm}_{S+}$ defined by a cd-structure, denoted by $\amalg$, generated by squares

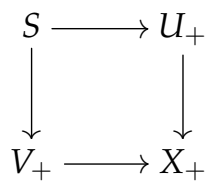

where $U$ and $V$ are clopen subschemes of $X$ such that $U \amalg V=X$. Sheaves with respect to the topology generated by this cd-structure is precisely the nonabelian derived category on $C$. In other words we have

$$
\operatorname{Shv}_{\amalg}\left(\mathrm{Sm}_{S+}\right) \simeq \mathrm{P}_{\Sigma}\left(\mathrm{Sm}_{S_{+}}\right)
$$


by [5, Lemma 2.4]. Hence all topologies $\tau$ considered in this paper satisfy $\operatorname{Shv}_{\tau}\left(\mathrm{Sm}_{S+}\right) \subseteq \mathrm{P}_{\Sigma}\left(\mathrm{Sm}_{S_{+}}\right)$.

Definition 4.8. Let $\tau$ be a topology on $S m_{S}$, and let $C$ be a correspondence category with graph functor $\gamma_{C}: \mathrm{Sm}_{S+} \rightarrow \mathrm{C}$. Then $\mathrm{C}$ is compatible with $\tau$ if for every $\tau$-sieve $U \hookrightarrow X$ in $\mathrm{Sm}_{S}$, the natural map

$$
\gamma_{\mathrm{C}}\left(U_{+}\right) \rightarrow \gamma_{\mathrm{C}}\left(X_{+}\right)
$$

is an $\mathrm{L}_{\tau}$-equivalence in $\mathrm{P}_{\Sigma}\left(\mathrm{Sm}_{S+}\right)$.

Lemma 4.9. Suppose that $\mathrm{C}$ is a correspondence category which is compatible with $\tau$. Then the functor

$$
\gamma_{C}: \mathrm{P}_{\Sigma}\left(\mathrm{Sm}_{S+}\right) \rightarrow \mathrm{P}_{\Sigma}\left(\mathrm{Sm}_{S+}\right)
$$

preserves $\mathrm{L}_{\tau}$-equivalences.

Proof. By definition, the class of $\mathrm{L}_{\tau}$-equivalences is the strong saturation, in the sense of [29, Proposition 5.5.4.5], of the maps in $\mathrm{P}_{\Sigma}\left(\mathrm{Sm}_{S+}\right)$ by the (Yoneda image of the) maps $i_{+}: U_{+} \hookrightarrow X_{+}$where $X \in \mathrm{Sm}_{S}$ and $i$ is a $\tau$-sieve. According to [5, Lemma 2.10], the class of $\mathrm{L}_{\tau}$-equivalences is then generated under 2-out-of-3 and sifted colimits by maps of the form $\pi_{X} \amalg \mathrm{id}_{Y_{+}}$for $Y \in \mathrm{Sm}_{S}$. By the same reasoning as in Proposition 4.7 we need only check that $\gamma_{C}\left(U_{+}\right) \rightarrow \gamma_{C}\left(X_{+}\right)$is an $L_{\tau}$-equivalence which is true by hypothesis.

From now on, whenever we consider a correspondence category $\mathrm{C}$ we make the following assumption on the topologies we discuss:

- The topology $\tau$ is at least as fine as the Nisnevich topology and is compatible in the sense of Definition 4.8 .

4.0.6. If $\mathrm{C}$ is a correspondence category, then we can construct its unstable motivic homotopy $\infty$-category in the usual way, as we now do. We consider two full subcategories of $\mathrm{P}_{\Sigma}(\mathrm{C})$ spanned by objects $\mathscr{F}$ satisfying the following two axioms on homotopy invariance and $\tau$-descent:

(Htpy): The presheaf $\mathscr{F} \circ \gamma_{C}: \mathrm{Sm}_{S}^{\text {op }} \rightarrow \mathrm{Spc}$ is $\mathrm{A}^{1}$-invariant. We denote the $\infty$-category spanned by such $\mathscr{F}^{\prime}$ 's by $\mathrm{P}_{\mathrm{A}^{1}}(\mathrm{C})$.

( $\tau$-Desc): The presheaf $\mathscr{F} \circ \gamma_{C}: \mathrm{Sm}_{S}^{\mathrm{op}} \rightarrow \mathrm{Spc}$ is a $\tau$-sheaf. We denote the $\infty$-category spanned by such $\mathscr{F}^{\prime}$ s by $\operatorname{Shv}_{\tau}(\mathrm{C})$.

Since $\mathrm{P}_{\Sigma}(\mathrm{C})$ is preadditive by Proposition (4.5), we have a canonical equivalence $C \operatorname{Mon}\left(\mathrm{P}_{\Sigma}(\mathrm{C})\right) \simeq \mathrm{P}_{\Sigma}(\mathrm{C})$. The $\infty$-category of unstable $C$-motives, denoted by $\mathrm{H}_{\tau}(\mathrm{C})$, is then defined as $\mathrm{P}_{\mathbf{A}^{1}}(\mathrm{C}) \cap \operatorname{Shv}_{\tau}(\mathrm{C}) \subseteq \mathrm{P}_{\Sigma}(\mathrm{C})$. As usual we have localization functors $\mathrm{L}_{\tau}^{C}: \mathrm{P}_{\Sigma}(\mathrm{C}) \rightarrow \operatorname{Shv}_{\tau}(\mathrm{C}), \mathrm{L}_{\mathrm{A}^{1}}^{\mathrm{C}}: \mathrm{P}_{\Sigma}(\mathrm{C}) \rightarrow \mathrm{P}_{\mathrm{A}^{1}}(\mathrm{C})$ and $\mathrm{L}_{\text {mot }, \tau}^{\mathrm{C}}: \mathrm{P}_{\Sigma}(\mathrm{C}) \rightarrow$ $\mathrm{H}_{\tau}(\mathrm{C})$. From the construction of these localizations and the assumption on $\tau$, the adjunction (4.3) descends to an adjunction

$$
\gamma_{C}^{*}: \mathrm{H}_{\tau}\left(\mathrm{Sm}_{S+}\right) \simeq \mathrm{H}_{\tau}(S)_{*} \rightleftarrows \mathrm{H}_{\tau}(\mathrm{C}): \gamma_{C *}
$$

Lemma 4.10. The $\infty$-category $\mathrm{H}_{\tau}(\mathrm{C})$ is preadditive. Hence we have a canonical equivalence $\mathrm{CMon}\left(\mathrm{H}_{\tau}(\mathrm{C})^{\times}\right) \simeq$ $\mathrm{H}_{\tau}(\mathrm{C})$.

Proof. The $\infty$-category $\mathrm{H}_{\tau}(\mathrm{C})$ is closed under finite products by checking that the conditions (Htpy) and ( $\tau$-Desc) are preserved under taking products which are computed pointwise. The statement follows since $\mathrm{P}_{\Sigma}(\mathrm{C})$ is preadditive by Proposition 4.5.

Definition 4.11. The $\infty$-category of effective $\mathrm{C}$-motives $\mathrm{H}_{\tau}(\mathrm{C})^{\mathrm{gP}}$ is defined to be the full subcategory of $\mathrm{H}_{\tau}(\mathrm{C})$ spanned by the grouplike objects, in the sense of [21, Definition 1.2].

4.0.7. The next proposition captures the main property of categories of correspondences from the point of view of motivic homotopy theory.

Proposition 4.12. Suppose that $\mathrm{C}$ is a correspondence category which is compatible with $\tau$. Then the functor

$$
\gamma_{C *}: \mathrm{H}_{\tau}(\mathrm{C}) \rightarrow \mathrm{H}_{\tau}(S)_{*}
$$

preserves sifted colimits and is conservative. Furthermore, $\mathrm{H}_{\tau}(\mathrm{C})$ is canonically an $\mathrm{H}(S)_{*}$-module. 
Proof. For the first claim it suffices, after Proposition 4.5, to check that

$$
\gamma_{\mathrm{C} *}: \mathrm{P}_{\Sigma}(\mathrm{C}) \rightarrow \mathrm{P}_{\Sigma}\left(\mathrm{Sm}_{\mathrm{S}+}\right) \simeq \mathrm{P}_{\Sigma}\left(\mathrm{Sm}_{S}\right)_{*}
$$

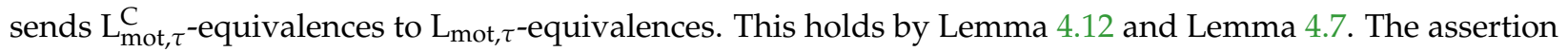
that $\mathrm{H}_{\tau}(\mathrm{C})$ is an $\mathrm{H}(S)_{*}$-module follows from the right lax structure of $\gamma_{C *}$.

Remark 4.13. If $\tau$ is a topology finer than the Nisnevich topology, then the fully faithful functor $\mathrm{H}_{\tau}(S)_{*} \rightarrow$ $\mathrm{H}(S)_{*}$ need not preserve colimits. Hence the composite $\mathrm{H}_{\tau}(C) \rightarrow \mathrm{H}_{\tau}(S)$ need not preserve colimits.

4.0.8. From the above point of view, we see that $\gamma_{C *}$ is very close to preserving all colimits-we need only show that it preserves finite coproducts. The universal way to enforce this is to take commutative monoid objects on both sides with respect to Cartesian monoidal structures. We can do this for $\mathrm{H}_{\tau}(S)_{*}$ since it has finite products, and $\mathrm{CMon}\left(\mathrm{H}_{\tau}(\mathrm{C})^{\times}\right) \simeq \mathrm{H}_{\tau}(\mathrm{C})$ since it is preadditive [21, Proposition 2.3]. We remark that the symmetric monoidal structure on $\mathrm{P}_{\Sigma}\left(\mathrm{Sm}_{S+}\right)$ given by Day convolution is not Cartesian. ${ }^{5}$

To see this, consider the left adjoint to $\gamma_{C_{*}}$, that is,

$$
\gamma_{C}^{*}: \mathrm{H}_{\tau}(S)_{*} \rightarrow \mathrm{H}_{\tau}(\mathrm{C}),
$$

which preserves all small colimits. According to the universal property of CMon [21, Corollary 4.9] we obtain an essentially unique functor $\gamma_{\mathrm{C}}^{*}: \mathrm{CMon}\left(\mathrm{H}_{\tau}(S)_{*}^{\times}\right) \rightarrow \mathrm{H}_{\tau}(\mathrm{C})$ since $\mathrm{H}_{\tau}(\mathrm{C})$ is preadditive by Proposition 4.5.1. This functor admits a right adjoint $\gamma_{C *}: \mathrm{H}_{\tau}(\mathrm{C}) \rightarrow \mathrm{CMon}\left(\mathrm{H}_{\tau}(S)_{*}^{\times}\right)$which fits into a commutative diagram

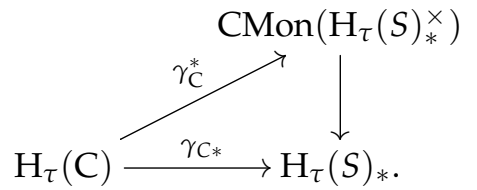

In other words, the functor $\gamma_{C *}$ factors through the forgetful functor $\mathrm{CMon}\left(\mathrm{H}_{\tau}(S)_{*}^{\times}\right) \rightarrow \mathrm{H}_{\tau}(S)_{*}$.

Proposition 4.14. Suppose that $\mathrm{C}$ is a correspondence category which is compatible with $\tau$. Then the functor

$$
\gamma_{C *}: \mathrm{H}_{\tau}(\mathrm{C}) \rightarrow \operatorname{CMon}\left(\mathrm{H}_{\tau}(S)_{*}^{\times}\right)
$$

preserves all small colimits and is conservative.

Proof. By the diagram (4.5), the functor $\gamma_{C *}$ preserves sifted colimits because the horizontal arrow preserves sifted colimits by Proposition 4.12 and the vertical arrow preserves sifted colimits as a special case of [21, Proposition B.4]. Since it is a right adjoint it preserves finite products, but since its domain and codomain are preadditive it preserves finite coproducts as well and we are done by [5, Lemma 2.8]. The conservativity statement follows from Proposition 4.12 and the fact that the forgetful functor from commutative monoid objects is conservative.

4.0.9. $\mathbb{T}$-stability. We now introduce the notion of $\mathbb{T}$-stability along with the weaker notion of $\mathbb{T}$-prestability. This is inspired by the treatement of [31, Appendix C] on prestable $\infty$-categories.

Definition 4.15. Let $\mathrm{C}$ be an $\mathrm{H}(S)_{*}$-module in $\mathrm{Cat}_{\infty}$. Then $\mathrm{C}$ is $\mathbb{T}$-prestable if the endofunctor

$$
\mathbb{T} \otimes(-): C \rightarrow C
$$

is fully faithful. The $\infty$-category $\mathrm{C}$ is $\mathbb{T}$-stable if the endofunctor (4.6) is invertible.

Remark 4.16. The notion of a $\mathbb{T}$-stable $\infty$-category is a familiar one in motivic homotopy theory; indeed, the motivic stable homotopy category $\mathrm{SH}(S)$ is $\mathbb{T}$-stable. In fact, $\mathbb{T}$-prestability is a familiar concept as well: it is inspired by Voevodsky's cancellation theorem [41] which asserts that $\mathrm{DM}^{\mathrm{eff}}(k ; \mathbf{Z})$ is $\mathbb{T}$-prestable for any perfect field $k$. The analogous statement holds for Milnor-Witt motivic cohomology as proved in [20]. For the $\infty$-category of framed motivic spaces, cancellation holds by [12, Theorem 3.5.8], which in turn relies on the cancellation theorem of Ananyevskiy, Garkusha and Panin [1]. Moreover, for any base scheme $S$, the subcategory $\mathrm{SH}(S)^{\text {eff }} \subseteq \mathrm{SH}(S)$ of effective motivic spectra is $\mathbb{T}$-prestable.

\footnotetext{
${ }^{5}$ On the other hand, the symmetric monoidal structure on $\mathrm{P}_{\Sigma}\left(\mathrm{Sm}_{S}\right)$ given by Day convolution is Cartesian, and the natural sifted-colimit preserving functor $\mathrm{P}_{\Sigma}\left(\mathrm{Sm}_{S}\right) \rightarrow \mathrm{P}_{\Sigma}\left(\mathrm{Sm}_{S+}\right)$ is symmetric monoidal.
} 
4.0.10. The thesis of Robalo [36] provides a way to invert $\mathbb{T}$ for any $\mathrm{H}(C)_{*}$-module and obtain a symmetric monoidal stable $\infty$-category-in fact one that is a module over $\mathrm{SH}(S)$. We define the stable $\infty$-category of C-motives simply by

$$
\mathrm{SH}_{\tau}(\mathrm{C}):=\mathrm{H}_{\tau}(\mathrm{C})\left[\mathrm{T}^{\otimes-1}\right],
$$

with notation as in [36, Definition 2.6]. We then have the basic adjunction

$$
\Sigma_{\mathbb{T}, \mathrm{C}}^{\infty}: \mathrm{H}_{\tau}(\mathrm{C}) \rightleftarrows \mathrm{SH}_{\tau}(\mathrm{C}): \Omega_{\mathbb{T}, \mathrm{C}}^{\infty} .
$$

The following summarizes the basic properties of $\mathrm{SH}_{\tau}(\mathrm{C})$ :

Proposition 4.17. If $\mathrm{C}$ is a correspondence category, then the following hold:

(1) The $\infty$-category $\mathrm{SH}_{\tau}(\mathrm{C})$ is a presentably symmetric monoidal stable $\infty$-category, and

(2) is generated under sifted colimits by objects of the form $\left\{\mathbb{T}^{\otimes n} \otimes \Sigma_{\mathbb{T}, C}^{\infty} X\right\}_{n \in \mathbf{Z}, X \in C}$.

(3) The $\infty$-category $\mathrm{SH}_{\tau}(\mathrm{C})$ is computed as the colimit in $\operatorname{Mod}_{\mathrm{H}\left(\mathrm{Sm}_{S}\right)_{*}}\left(\operatorname{Pr}^{L}\right)$ of

$$
\mathrm{H}_{\tau}(C) \stackrel{\mathbb{T} \otimes(-)}{\longrightarrow} \mathrm{H}_{\tau}(\mathrm{C}) \stackrel{\mathbb{T} \otimes(-)}{\longrightarrow} \mathrm{H}_{\tau}(\mathrm{C}) \stackrel{\mathbb{T} \otimes(-)}{\longrightarrow} \cdots .
$$

(4) The functor

is conservative and preserves colimits.

$$
\gamma_{C *}: \mathrm{SH}_{\tau}(\mathrm{C}) \rightarrow \mathrm{SH}_{\tau}\left(\mathrm{Sm}_{S}\right)
$$

Proof. Stability follows from the standard equivalence $\mathbb{T} \simeq S^{1} \otimes \mathbf{G}_{m}$ in $\mathrm{SH}(S)$, which remains true for modules over $\mathrm{SH}(S)$. The second assertion follows from the third via [29, Lemma 6.3.3.7] and the fact that $\mathrm{H}_{\tau}(\mathrm{C})$ is generated under sifted colimits by representables which are smooth affine by the argument of [27, Proposition 2.2.9] (which works for any topology $\tau$ finer than Nis), while the third comes from [36, Corollary 2.22]. The last assertion follows from Proposition 4.14.

4.0.11. The last part of Proposition 4.17 is the main point of our axiomatization: the adjunction $\mathrm{SH}_{\tau}(S) \rightleftarrows$ $\mathrm{SH}_{\tau}(C)$ is monadic. In particular, if $\tau=$ Nis, then $\mathrm{SH}(S) \rightleftarrows \mathrm{SH}(C)$ is monadic.

4.1. From categories of correspondences to motivic module categories. Suppose that we have a functor

$$
\mathrm{C}: \mathscr{S}^{\mathrm{op}} \rightarrow \text { CorrCat }^{\otimes}
$$

which carries a morphism of schemes $f: T \rightarrow S$ to $f^{*}: C_{S} \rightarrow C_{T}$. By naturality of the preceding constructions ${ }^{6}$ we obtain a functor

$$
\mathrm{SH}_{\tau} \circ \mathrm{C}: \mathscr{S}^{\mathrm{op}} \rightarrow \operatorname{Pr}_{\text {stab }}^{L, \otimes}
$$

equipped with a transformation $\left.\mathrm{SH}\right|_{\mathscr{S}} \rightarrow \mathrm{SH}_{\tau} \circ \mathrm{C}$. We impose an additional assumption on $\mathrm{C}$, inspired by [7, Lemma 9.3.7]:

- For each $p: T \rightarrow S$, a smooth morphism in $\mathscr{S}$, the functor $p^{*}$ admits a left adjoint $p_{\#}$ such that the transformation $p_{\#} \gamma_{C_{T}} \rightarrow \gamma_{C_{S}} p_{\#}$ is an equivalence.

In this case, we say that $C$ is adequate.

4.1.1. We employ the following additional notation: if $L: \mathrm{SH}(S) \rightarrow \mathrm{SH}(S)$ is a localization, denote by $L\left(\mathrm{SH}_{\tau}\left(\mathrm{C}_{S}\right)\right)$ the subcategory of $\mathrm{SH}_{\tau}\left(\mathrm{C}_{S}\right)$ spanned by objects $X$ such that $\gamma_{C *} X$ is $L$-local. Since $\gamma_{C *}$ preserves limits, the inclusion $L\left(\mathrm{SH}_{\tau}\left(\mathrm{C}_{S}\right)\right) \hookrightarrow \mathrm{SH}_{\tau}\left(\mathrm{C}_{S}\right)$ is closed under limits and there is a localization functor (by the adjoint functor theorem)

$$
L_{\mathrm{C}_{S}}: \mathrm{SH}_{\tau}\left(\mathrm{C}_{S}\right) \rightarrow L\left(\mathrm{SH}_{\tau}\left(\mathrm{C}_{S}\right)\right)
$$

rendering the following diagram commutative (since their right adjoints commute):

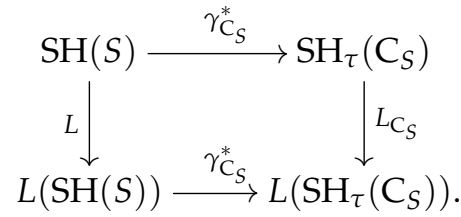

Proposition 4.18. If C: $\mathscr{S}^{\mathrm{op}} \rightarrow \mathrm{CorrCat}^{\otimes}$ is adequate, then the following hold:

\footnotetext{
${ }^{6}$ The most nontrivial of which is the universal property of $\mathbb{T}$-stabilization for which we can appeal to [5, Lemma 4.1].
} 
(1) We have premotivic adjunctions $\left.\mathrm{SH}\right|_{\mathscr{S}} \rightleftarrows \mathrm{SH}_{\tau} \circ \mathrm{C}$.

(2) If $L$ is smashing and a symmetric monoidal localization of $\left.\mathrm{SH}\right|_{\mathscr{S}}$, then we have a premotivic adjunction $\left.L(\mathrm{SH})\right|_{\mathscr{S}} \leftrightarrows L(\mathrm{SH} \circ \mathrm{C})$.

(3) If $\tau$ is a topology such that for each $S \in \mathscr{S}$, the functor $L\left(\mathrm{SH}_{\tau}(S)\right) \rightarrow L(\mathrm{SH}(S))$ preserves sifted colimits, then the premotivic adjunction $\left.L(\mathrm{SH})\right|_{\mathscr{S}} \rightleftarrows L\left(\mathrm{SH}_{\tau} \circ \mathrm{C}\right)$ is a motivic module category (in particular, this holds when $\tau=$ Nis).

Proof. The proof of (1) follows as in the case of Grothendieck abelian categories [7, Corollary 10.3.11] and Voevodsky's $C=$ Corr (in the sense of $[7, \S 9]$ ); we give only the main points. Since $C$ is adequate, we get that the equivalence $p_{\#} \gamma_{C_{T}} \rightarrow \gamma_{C} p_{\#}$ persists on the level of $\mathbb{T}$-stabilizations. What we need to verify, just as in [7, Proposition 10.3.9], is that the transformation $L_{\tau} \gamma_{C *} \simeq \gamma_{C *} L_{\tau}$ is an equivalence on the unstable level, i.e., the "forgetful" functor $\left.\mathrm{H}_{\tau} \circ \mathrm{C} \rightarrow \mathrm{H}\right|_{\mathscr{S}}$ preserves $\tau$-local objects. This is given by Lemma 4.9 under the standing assumption that $C$ is compatible with $\tau$. The next two statements are then immediate from the definition of motivic module categories and the last statement of Proposition 4.17.

4.2. Examples. We now discuss examples of the above constructions and results.

Example 4.19. Let $\mathscr{S}=\mathrm{Sch}_{S}$ and suppose that $\mathrm{E}$ is an $\mathcal{E}_{\infty}$-ring spectrum in $\mathrm{SH}(S)$. Then $\operatorname{Mod}_{\mathrm{E}}=(\mathrm{E} \otimes(-)) \circ$ $\mathrm{SH}$ furnishes the first examples of motivic module categories. We can also consider further localizations of the premotivic category $\operatorname{Mod}_{E}$, such as in [19] where $\mathscr{S}=\operatorname{Sch}_{\mathbb{Z}\left[\frac{1}{\ell}\right]}$ the localization functor is given by the composite of $\ell$-completion and étale localization, and $\mathrm{E}$ is MGL; see loc. cit. for more details where results in this paper is used to describe the $\infty$-category of modules over étale cobordism.

Example 4.20. Consider a localization $L:\left.\mathrm{SH}\right|_{\mathscr{S}} \rightarrow L\left(\left.\mathrm{SH}\right|_{\mathscr{S}}\right)$. If $L$ is smashing, then $L\left(\left.\mathrm{SH}\right|_{\mathscr{S}}\right)$ is a motivic module category. Examples of these smashing localizations are given by periodization of elements; we refer the reader to [23, Section 3] for an extensive discussion in our context. For example, a theorem of Bachmann [3] proves that periodizing the element $\rho$ yields real étale localization. Consider $x: \Sigma^{p, q} \mathbf{1} \rightarrow \mathbf{1}$. Then the results of $[23, \S 3]$ (or apply [3, Lemma 15]) tell us that $\mathbf{1}\left[x^{-1}\right]$ is an $\mathcal{E}_{\infty}$-ring and the projection formula holds, hence the category of $x$-periodic motivic spectra are modules over $\mathbf{1}\left[x^{-1}\right]$.

Example 4.21. The basic example of a category of correspondences is Voevodsky's category of finite correspondences Corr in $_{S}$ the sense of [33, Appendix 1A] [7, §9], which is defined for any Noetherian scheme $S[7, \S 9.1]$. When $S$ is essentially smooth over a perfect base field, the category of finite Milnor-Witt correspondences $\widetilde{\text { Corr }_{S}}$ of Calmès and Fasel [6] is defined and is also a category of correspondences. Over a perfect field (where both categories are defined), these categories are generalized by Garkusha's axioms in [16]. When defined, these categories are adequate in the sense of $\S 4$.1. All of these are examples of categories of correspondences, and thus gives rise motivic module categories.

Example 4.22. Let $k$ be a perfect field. Given any $S \in \mathrm{Sm}_{k}$ and any good cohomology theory $A$ on $\mathrm{Sm}_{S}$ in the sense of [11, §2], then [11, §3] defines an adequate category of correspondences $\operatorname{Corr}_{S}^{A}$ on $\mathrm{Sm}_{S}$.

Example 4.23. The $\infty$-category of framed correspondences of [12] is another example of a category of correspondences and is defined for any qcqs scheme $S$. The main theorem of [24] asserts that the corresponding motivic module category is equivalent to $\mathrm{SH}(S)$, relying on the "recognition principle" of [12].

Example 4.24. If $\mathrm{E} \in \mathrm{SH}(S)$ is a homotopy associative ring spectrum, then [13] defines a hSpc-enriched category $\mathrm{hCorr} \mathrm{E}_{S}^{\mathrm{E}}$ of finite $\mathrm{E}$-correspondences, which the authors expect to be the homotopy category of an $\infty$-category Corr ${ }_{S}^{\mathrm{E}}$ whenever $\mathrm{E}$ is an $\mathcal{A}_{\infty}$-ring. Setting $\mathrm{C}=\operatorname{Corr}_{S}^{\mathrm{E}}$, the $\infty$-category $\mathrm{SH}(\mathrm{C})$ in this paper corresponds to $\mathrm{DM}^{\mathrm{E}}(S)$ in loc. cit. We will return to this example in the next section.

\section{MODULE CATEGORIES OVER REGULAR SCHEMES}

In this section we show that the hypotheses of Theorem 3.5 are satisfied for module categories over a field $k$, and more generally for module categories over regular $k$-schemes.

5.1. The case of fields. We start by verifying that the projection formula holds at a field $k$. In this case, we can use the following computation to reduce to the case of compact-rigid generation: 
Lemma 5.1. Suppose that we have an adjunction of symmetric monoidal $\infty$-categories

$$
F: \mathrm{C} \rightleftarrows \mathrm{D}: \mathrm{G}
$$

such that $\mathrm{F}$ is strongly symmetric monoidal. Let $1 \in \mathrm{C}$ denote the unit object of $\mathrm{C}$. If $\mathrm{E} \in \mathrm{C}$ is a strongly dualizable object, then the map c: $G F(1) \otimes \mathrm{E} \rightarrow G F(\mathrm{E})$ is an equivalence.

Proof. This follows from a standard computation: let $\mathrm{E}^{\prime} \in \mathrm{C}$ be arbitrary, then we have a string of equivalences

$$
\begin{aligned}
\operatorname{Maps}_{\mathrm{C}}\left(\mathrm{E}^{\prime}, G F(1) \otimes \mathrm{E}\right) & \simeq \operatorname{Maps}_{\mathrm{C}}\left(\mathrm{E}^{\prime} \otimes \mathrm{E}^{\vee}, G F(1)\right) \\
& \simeq \operatorname{Maps}_{\mathrm{D}}\left(F\left(\mathrm{E}^{\prime} \otimes \mathrm{E}^{\vee}\right), F(1)\right) \\
& \simeq \operatorname{Maps}_{\mathrm{D}}\left(F\left(\mathrm{E}^{\prime}\right) \otimes F(\mathrm{E}){ }^{\vee}, F(1)\right) \\
& \simeq \operatorname{Maps}_{\mathrm{D}}\left(F\left(\mathrm{E}^{\prime}\right), F(\mathrm{E})\right) \\
& \simeq \operatorname{Maps}_{\mathrm{C}}\left(\mathrm{E}^{\prime}, G F(\mathrm{E})\right),
\end{aligned}
$$

which shows the claim.

5.1.1. Thus, if $\mathrm{SH}(S)$ is generated by strongly dualizable objects, it follows that the projection formula holds:

Theorem 5.2. Let $k$ be a field. Suppose that $\ell$ is a prime which is coprime to the exponential characteristic e of $k$ and let $\mathscr{M}$ be a motivic module category on $k$. Then we have the following equivalences of presentably symmetric monoidal stable $\infty$-categories:

and

$$
L_{(\ell)}(\mathscr{M}(k)) \simeq \operatorname{Mod}_{L_{(\ell)} \gamma_{*} \gamma^{*}\left(\mathbf{1}_{S}\right)}(\operatorname{SH}(k)),
$$

$$
\mathscr{M}(k)\left[\frac{1}{e}\right] \simeq \operatorname{Mod}_{\gamma_{*} \gamma^{*}(\mathbf{1})\left[\frac{1}{e}\right]}(\mathrm{SH}(k)) .
$$

Proof. In light of Theorem 3.5 we need to verify the appropriate projection formulas. By assumption, the functor $\gamma_{*}$ preserves sifted colimits and thus the functors $\gamma_{*} \gamma^{*}\left(\mathbf{1}_{S}\right) \otimes(-)$ and $\gamma_{*} \gamma^{*}(-)$ do as well. Now Lemma 5.1 tells us that the projection formula holds for strongly dualizable objects in $\operatorname{SH}(k)_{(\ell)}$. Thus we will be done if we can prove that the inclusion of (2.1), $\mathrm{SH}^{\mathrm{rig}}(k)_{(\ell)} \subseteq \mathrm{SH}^{\omega}(k)_{(\ell)}$, is an equality. This amounts to showing that $\mathrm{SH}(k)_{(\ell)}$ is in fact generated by sifted colimits by strongly dualizable objects. But this follows by Lemma 2.2, which also verifies the theorem for the $e$-inverted case.

5.1.2. We now obtain the following extension of [38, Theorem 1], [25, Theorem 5.8], [16, Theorem 5.3] and [4, Lemma 5.3]:

Corollary 5.3. Let $k$ be a field of exponential characteristic e and let $\gamma_{C}: \mathrm{Sm}_{k} \rightarrow \mathrm{C}$ be a correspondence category over $k$. Then there is an equivalence of presentably symmetric monoidal stable $\infty$-categories

$$
\mathrm{SH}(\mathrm{C})\left[\frac{1}{e}\right] \simeq \operatorname{Mod}_{\gamma_{C *} \gamma_{C}^{*}(\mathbf{1})\left[\frac{1}{e}\right]}(\mathrm{SH}(k)) .
$$

5.2. The case $\mathscr{S}=\operatorname{Reg}_{k}$. Following [9] we can extend the previous result to the category $\operatorname{Reg}_{k}$ of finite dimensional Noetherian schemes that are regular over a field $k$, provided that we impose some additional assumptions on $\mathscr{M}$. For the rest of this section, we will therefore assume that $\mathscr{M}$ is a motivic module category which in addition satisfies the following property:

- The premotivic category $\mathscr{M}$ satisfies localization (Definition 2.5) and continuity (Definition 2.6).

Lemma 5.4. Suppose that $f: T \rightarrow S$ is a morphism in $\operatorname{Reg}_{k}$. In the following cases, the transformation

$$
f^{*} \gamma_{*} \rightarrow \gamma_{*} f^{*}
$$

is an equivalence:

(1) The scheme $T$ is an inverse limit $\lim _{\alpha} T_{\alpha}$ of $S$-schemes $T_{\alpha}$ such that the transition maps $T_{\alpha} \rightarrow T_{\beta}$ are dominant, affine and smooth.

(2) The map $f$ is a closed immersion and $S \cong \lim _{\alpha} S_{\alpha}$, where each $S_{\alpha}$ is a smooth, separated $k$-scheme of finite type with flat affine transition maps. 
Proof. Under the continuity and localization assumption on $\mathscr{M}$, the proof in [9, Lemma 3.20] for the case of $\mathscr{M}=\mathrm{DM}(-; R)$ applies verbatim.

5.2.1. We now have the following extension of Theorem 5.2.

Theorem 5.5. Let $k$ be a field of exponential characteristic e, and let $\mathscr{M}$ be a motivic module category on $\operatorname{Reg}_{k}$. Then the functor $\gamma^{*}: \mathrm{SH} \rightarrow \mathscr{M}$ induces a canonical equivalence

$$
\operatorname{Mod}_{\gamma_{*} \gamma^{*}(\mathbf{1})\left[\frac{1}{e}\right]}(\mathrm{SH}(-)) \stackrel{\simeq}{\rightarrow} \mathscr{M}\left[\frac{1}{e}\right]
$$

of premotivic categories on $\operatorname{Reg}_{k}$.

Proof. After Theorem 3.5, our goal is to verify that $\left(\left.\mathrm{SH}\right|_{\operatorname{Reg}_{k}}, \mathscr{M}\right)$ satisfies the projection formula. Suppose that $S \in \operatorname{Reg}_{k}$, and let $\mathrm{E} \in \mathrm{SH}(S)$. We claim that the map

$$
\gamma_{*} \gamma^{*}\left(\mathbf{1}_{S}\right) \otimes \mathrm{E} \rightarrow \gamma_{*} \gamma^{*}(\mathrm{E})
$$

is an equivalence. To show this, we follow closely the logic of [9, Theorem 3.1].

First, assume that $S$ is an essentially smooth scheme over a field. For each $x \in S$, we write $S_{x}$ for the localization of $S$ at $x$. Then the family of functors

$$
\left\{\mathrm{SH}(S) \rightarrow \mathrm{SH}\left(S_{x}\right)\right\}
$$

is conservative by [7, Proposition 4.3.9]. Hence we are reduced to proving that the map (5.1) is an equivalence in the case $S$ is furthermore local. In this case, let $i: x \hookrightarrow S_{x}$ be the closed point and write $j: U_{x} \rightarrow S_{x}$ for the open complement. By our assumption on $S, U_{x}$ has dimension $<\operatorname{dim} S$. We consider the following commutative diagram, where the rows are cofiber sequences:

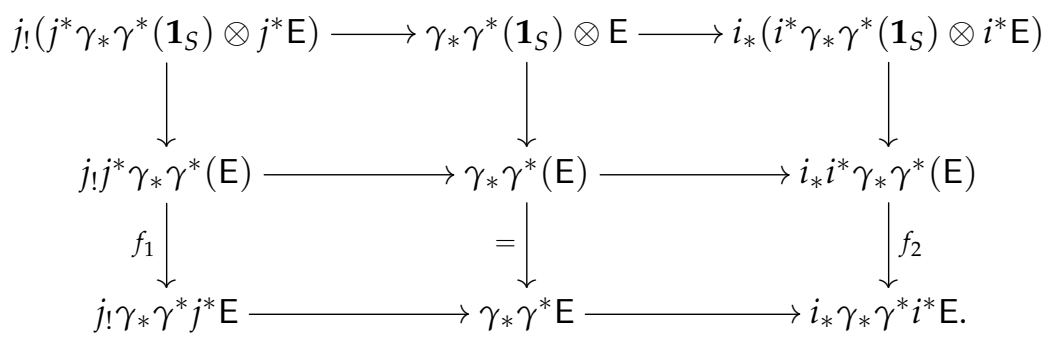

Now,

- The left vertical composite is an equivalence because (1) $j_{*}$ commutes with $\gamma_{*}$ by definition of a morphism of premotivic categories, and (2) by the induction hypothesis.

- The right vertical composite is an equivalence using (1) Lemma 5.4.2 and (2) the case of fields, Theorem 5.2.

It therefore remains to show that $f_{1}$ and $f_{2}$ are equivalences.

- The map $f_{1}$ is an equivalence because $j_{*}$ commutes with $\gamma_{*}$.

- That $f_{2}$ is an equivalence follows from Lemma 5.4.2.

Now, following the "General case" of [9], we explain how the bootstrap to regular k-schemes work. By continuity (appealing to [7, Proposition 4.3.9] again), we may again assume that $S$ is a Henselian local regular $k$-scheme. As explained in loc. cit., there is a sequence of regular Noetherian $k$-schemes

$$
T \stackrel{f}{\rightarrow} S^{\prime} \stackrel{q}{\rightarrow} S
$$

such that the following hold:

- The scheme $S^{\prime}$ has infinite residue field and the functor $q^{*}: \mathrm{SH}(S)\left[\frac{1}{e}\right] \rightarrow \mathrm{SH}\left(S^{\prime}\right)\left[\frac{1}{e}\right]$ is conservative.

- The scheme $T$ is the $\infty$-gonflement of $\Gamma\left(S^{\prime}, \mathscr{O}_{S^{\prime}}\right)\left[9\right.$, Definition 3.21] and the functor $f^{*}: \operatorname{SH}\left(S^{\prime}\right)\left[\frac{1}{e}\right] \rightarrow$ $\mathrm{SH}(T)\left[\frac{1}{e}\right]$ is conservative.

- Both $f$ and $q$ satisfy the hypotheses of Lemma 5.4.1, and thus $f^{*}$ and $q^{*}$ commute with $\gamma_{*}$. 
Hence, to check that the map (5.1) is an equivalence it suffices to check that it is an equivalence after applying $(q f)^{*}$. Since $T$ is, by construction, the spectrum of a filtered union of its smooth subalgebras we invoke continuity of $\mathrm{SH}$ to conclude.

5.2.2. Lastly, we provide the following class of examples of motivic module categories for which localization and continuity holds. We will make the following assumption:

- for a base scheme $S$ and an $\mathcal{A}_{\infty}$-ring spectrum $\mathrm{E} \in \mathrm{SH}(S)$, there exists an $\infty$-category Corr $\mathrm{E}_{S}^{\mathrm{E}}$ such that its homotopy category is the hSpc-enriched category hCorr ${ }_{S}^{\mathrm{E}}$ of [13].

With this assumption in play, any motivic $\mathcal{A}_{\infty}$-ring spectrum E gives rise to the motivic module category $\mathrm{DM}^{\mathrm{E}}$ as explained in Example 4.24 and [13]. While this makes the next results conditional, we will explain unconditional instances of these results in Example 5.9.

Proposition 5.6. Let $\mathscr{S} \subseteq \mathrm{Sch}_{S}$. Then, for any $\mathcal{A}_{\infty}$-ring spectrum $\mathrm{E} \in \mathrm{SH}(S)$, the premotivic category $\mathrm{DM}^{\mathrm{E}}: \mathscr{S}$ op $\rightarrow$ Cat $_{\infty}$ satisfies continuity for dominant affine morphisms.

Proof. We first claim the analog of [7, Proposition 9.3.9] for E-correspondences. Let $\left(X_{i}\right)_{i \in I}$ be a cofiltered diagram of separated $S$-schemes of finite type with affine dominant transition morphisms. Let $X=\lim _{i} X_{i}$, which is assumed to exist in $\mathrm{Sch}_{S}$ and is assumed to be Noetherian. Then we claim that for any separated $S$-scheme $Y$ of finite type, the map

$$
\underset{i \in I^{\mathrm{OP}}}{\operatorname{colim}} \operatorname{Corr}_{S}^{\mathrm{E}}\left(X_{i}, Y\right) \rightarrow \operatorname{Corr}_{S}^{\mathrm{E}}(X, Y)
$$

is an equivalence.

To do so, we use the dual of [12, Lemma 4.1.26]. Denote by $c_{X_{i}}$ (resp. $c_{X}$ ) the filtered poset of reduced subschemes of $X_{i} \times{ }_{S} Y\left(\right.$ resp. $\left.X \times{ }_{S} Y\right)$ which are finite and universally open over $X_{i}$ (resp. $\left.X\right)$. Furthermore, we denote by $\operatorname{Sub}\left(c_{X}\right)$ the poset of full sub-posets of $c_{X}$. We then have a functor $K: I \rightarrow \operatorname{Sub}\left(c_{X}\right)$ given by $i \mapsto K_{i}:=c_{X_{i}}$, where $c_{X_{i}}$ is regarded as a full sub-poset in the obvious way. By continuity of $\mathrm{SH}$, the functor $\mathrm{E}^{\mathrm{BM}}(-/ X): c_{X} \rightarrow$ Spc of Borel-Moore E-homology spaces $[13, \S 2]$ restricts to a functor $\mathrm{E}^{\mathrm{BM}}\left(-/ X_{i}\right): c_{X_{i}} \rightarrow$ Spc. Hence the map (5.3) is, by [13, Definition 4.1.1], equal to the map

$$
\underset{I^{\mathrm{OP}}}{\operatorname{colim}} \underset{c_{X_{i}}}{\operatorname{colim}} \mathrm{E}^{\mathrm{BM}}\left(Z_{i} / X_{i}\right) \rightarrow \underset{Z \in c_{X}}{\operatorname{colim}} \mathrm{E}^{\mathrm{BM}}(Z / X),
$$

which we claim is an equivalence. The hypotheses of [12, Lemma 4.1.26] follow easily (under the hypotheses that the transition maps are affine and dominant) by [7, Propositions 8.3.6, 8.3.9]. Hence the desired claim follows. The rest of the proof follows as in the case of DM from [7, Theorem 11.1.24].

Proposition 5.7. Let $k$ be a field and let $\mathrm{E} \in \mathrm{SH}(k)$ be an $\mathcal{A}_{\infty}$-ring spectrum. Then the premotivic category $\mathrm{DM}^{\mathrm{E}}: \mathscr{S}^{\mathrm{op}} \rightarrow$ Cat $_{\infty}$ satisfies $\left(\right.$ Loc $_{i}$ ) whenever $i$ is a closed immersion of regular schemes.

Proof. Since $\mathrm{DM}^{\mathrm{E}}$ is constructed from Nisnevich local objects, it is Nisnevich separated. By [7, Proposition 6.3.14], it has the weak localization property, i.e., it has $\left(\operatorname{Loc}_{i}\right)$ for any closed immersion with smooth retractions. Arguing as in [7, Corollary 6.3.15], it has the localization property with respect to any closed immersion between smooth schemes. The rest of the argument then follows as in [9, Proposition 3.12], which uses the continuity results established in Proposition 5.6 as above.

5.2.3. From this we conclude:

Corollary 5.8. Let $k$ be a field of exponential characteristic e and let $\mathrm{E} \in \mathrm{SH}(k)$ be an $\mathcal{A}_{\infty}$-ring spectrum. Then we have a canonical equivalence

$$
\mathrm{DM}^{\mathrm{E}}\left[\frac{1}{e}\right] \simeq \operatorname{Mod}_{\gamma_{*} \gamma^{*}(\mathbf{1})\left[\frac{1}{e}\right]}(\mathrm{SH}(-))
$$

of premotivic categories on $\operatorname{Reg}_{k}$.

Example 5.9. As explained in [13, 4.1.19], the hypothetical $\infty$-category $\operatorname{Corr} \mathrm{E}_{S}^{\mathrm{E}}$ is equivalent to hCorr $\mathrm{E}_{S}$ whenever $S$ is essentially smooth over a perfect field $k$ and $E$ is pulled back from the heart of the effective homotopy $t$-structure $\mathrm{SH}(k)^{\text {eff, },}$ over $k$. Hence Theorem 5.8 holds unconditionally whenever $E$ is pulled back from the prime subfield of $k$ and lies in the heart of the effective homotopy $t$-structure there. 
Examples of such spectra include the motivic cohomology spectrum HZ and its Milnor-Witt counterpart HZ̃. Furthermore, in [13, Proposition 4.3.6] (resp. [13, Proposition 4.3.19]) it is proved that $\mathrm{DM}^{\mathrm{HZ}}(S) \simeq$ $\operatorname{DM}(S)$ (resp. $\operatorname{DM}^{\mathrm{H} \widetilde{Z}}(S) \simeq \widetilde{\mathrm{DM}}(S)$ ) whenever $S$ is essentially smooth over a Dedekind domain (resp. essentially smooth over a perfect field) [13, Proposition 4.3.8] (resp. [13, Proposition 4.3.19]). By the continuity result of Proposition 5.6 we can enhance the comparison results for DM to regular schemes over fields. While $\widetilde{\mathrm{DM}}(S)$ is not defined outside of smooth schemes over perfect fields, Corollary 5.8 promotes the comparison results between $\widetilde{\mathrm{DM}}$ and modules over H $\widetilde{Z}$ of [16] and [4] at least to smooth schemes over fields. We contend, however, that $\mathrm{DM}^{\mathrm{H} \widetilde{Z}}(S)$ is a decent definition for $\widetilde{\mathrm{DM}}(S)$ in general.

\section{REFERENCES}

[1] A. Ananyevskiy, G. Garkusha, and I. Panin, Cancellation theorem for framed motives of algebraic varieties (2016), available at arXiv: 1601.06642. $\uparrow 4.16$

[2] J. Ayoub, Les six opérations de Grothendieck et le formalisme des cycles évanescents dans le monde motivique. I, Astérisque 314 (2007), $\mathrm{x}+466$ pp. (2008). $\uparrow 3.0 .1$

[3] T. Bachmann, Motivic and real étale stable homotopy theory, Compos. Math. 154 (2018), no. 5, 883-917, DOI 10.1112/S0010437X17007710. $\uparrow 4.20$

[4] T. Bachmann and J. Fasel, On the effectivity of spectra representing motivic cohomology theories (2017), available at arXiv: 1710.00594. $\uparrow 5.1 .2,5.9$

[5] T. Bachmann and M. Hoyois, Norms in motivic homotopy theory (2018), available at arXiv:1711.0306. $\uparrow 4,4.4,4.0 .4,4.0 .5,4.0 .5,4.0 .8$, 6

[6] B. Calmès and J. Fasel, The category of finite MW-correspondences (2017), available at arXiv:1412.2989v2. $\uparrow 4,4.21$

[7] D.-C. Cisinski and F. Déglise, Triangulated categories of mixed motives (2019), available at arXiv:0912.2110. To appear in Lecture Notes in Mathematics. $\uparrow 2.3,2.3 .1,3.0 .1,4.1,4.1 .1,4.21,5.2 .1,5.2 .1,5.2 .2,5.2 .2,5.2 .2$

[8] __ Mixed Weil cohomologies, Adv. Math. 230 (2012), no. 1, 55-130, DOI 10.1016/j.aim.2011.10.021. $\uparrow 2.3$

[9] __ Integral mixed motives in equal characteristic, Doc. Math. Extra vol.: Alexander S. Merkurjev's sixtieth birthday (2015), $145-194 . \uparrow 1,1,2.3 .2,5.2,5.2,5.2 .1,5.2 .1,5.2 .2$

[10] __Étale motives, Compos. Math. 152 (2016), no. 3, 556-666, DOI 10.1112/S0010437X15007459. $\uparrow 2.3,2.3 .1$

[11] A. Druzhinin and H. Kolderup, Cohomological correspondence categories (2019), available at arXiv: 1808.05803. $\uparrow 4,4.22$

[12] E. Elmanto, M. Hoyois, A. A. Khan, V. Sosnilo, and M. Yakerson, Motivic infinite loop spaces (2019), available at arXiv: 1711.05248. $\uparrow 4,4.16,4.23,5.2 .2$

[13] _ Framed transfers and motivic fundamental classes (2018), available at arXiv: 1809.10666. $\uparrow 4,4.24,5.2 .2,5.2 .2,5.9$

[14] E. Elmanto and A. A. Khan, Perfection in Motivic Homotopy Theory (2019), available at arXiv: 1812.07506. $\uparrow 2.2 .1$

[15] A. Druzhinin, Effective Grothendieck-Witt motives of smooth varieties (2017), available at arXiv:1709.06273. $\uparrow 4$

[16] G. Garkusha, Reconstructing rational stable motivic homotopy theory, Compos. Math. 155 (2019), no. 7, 1424-1443, DOI 10.1112/s0010437x19007425. $\uparrow 1,4.21,5.1 .2,5.9$

[17] F. Déglise and J. Fasel, MW-motivic complexes (2017), available at arXiv:1708.06095. $\uparrow 1,4$

[18] B. I. Dundas, O. Röndigs, and P. A. Østvær, Motivic functors, Doc. Math. 8 (2003), 489-525. $\uparrow 1$

[19] E. Elmanto, M. Levine, M. Spitzweck, and P. A. Østvær, Algebraic Cobordism and Étale Cohomology (2019), available at arXiv: 1711.06258. $\uparrow 4.19$

[20] J. Fasel and P. A. Østvær, A Cancellation Theorem for Milnor-Witt Correspondences (2017), available at arXiv: 1708.06098. $\uparrow 4.16$

[21] D. Gepner, M. Groth, and T. Nikolaus, Universality of multiplicative infinite loop space machines, Algebr. Geom. Topol. 15 (2015), no. 6, 3107-3153. $\uparrow 4.0 .2,4.11,4.0 .8,4.0 .8$

[22] M. Hoyois, The six operations in equivariant motivic homotopy theory, Adv. Math. 305 (2017), 197-279, DOI 10.1016/j.aim.2016.09.031. $\uparrow 2.2 .1$

[23] __ Equivariant classifying spaces and cdh descent for the homotopy K-theory of tame stacks (2017), available at arXiv: 1604.06410. $\uparrow 4.20$

[24] The localization theorem for framed motivic spaces (2018), available at arXiv: 1807.04253. $\uparrow 4.23$

[25] M. Hoyois, S. Kelly, and P. A. Østvær, The motivic Steenrod algebra in positive characteristic, J. Eur. Math. Soc. (JEMS) 19 (2017), no. 12, 3813-3849, DOI 10.4171/JEMS/754. $\uparrow 5.1 .2$

[26] D. Gaitsgory and N. Rozenblyum, A study in derived algebraic geometry. Vol. I. Correspondences and duality 221 (2017), xl+533pp. $\uparrow 2.1$

[27] A. Khan, Motivic homotopy theory in derived algebraic geometry, https://www . preschema. com/thesis/thesis.pdf. $\uparrow 2.2 .1,2.3,4.0 .10$

[28] M. Levine, Y. Yang, and G. Zhao, Algebraic elliptic cohomology theory and flops, I (2013), available at arXiv: 1311.2159. $\uparrow 2.2 .1$

[29] J. Lurie, Higher topos theory, Annals of Mathematics Studies, vol. 170, Princeton University Press, Princeton, NJ, 2009. MR2522659 (2010j:18001) $\uparrow 1.2,2.3 .1,4,4.0 .2,4.0 .4,4.0 .5,4.0 .10$

[30] __ Higher Algebra. September 18, 2017. Available at http://www.math.harvard.edu/ 1urie/papers/HA.pdf. $\uparrow 1,1.2,2.1$, 2.3.1, 3.7

[31] __ Spectral Algebraic Geometry. February 3, 2018. Available at http://www.math.harvard.edu/ lurie/papers/ SAG-rootfile.pdf. $\uparrow 4.0 .9$

[32] A. Mathew, N. Naumann, and J. Noel, Nilpotence and descent in equivariant stable homotopy theory, Adv. Math. 305 (2017), $994-1084$. $\uparrow 3.8$ 
[33] C. Mazza, V. Voevodsky, and C. Weibel, Lecture notes on motivic cohomology, Clay Mathematics Monographs, vol. 2, American Mathematical Society, Providence, RI; Clay Mathematics Institute, Cambridge, MA, 2006. $\uparrow 1,4,4.21$

[34] F. Morel and V. Voevodsky, $\mathbf{A}^{1}$-homotopy theory of schemes, Inst. Hautes Études Sci. Publ. Math. 90 (1999), 45-143. $\uparrow 4.0 .4$

[35] J. Riou, Dualité de Spanier-Whitehead en géométrie algébrique, C. R. Math. Acad. Sci. Paris 340 (2005), no. 6, 431-436, DOI 10.1016/j.crma.2005.02.002. $\uparrow 2.2 .1$

[36] M. Robalo, K-theory and the bridge from motives to noncommutative motives, Adv. Math. 269 (2015), 399-550, DOI 10.1016/j.aim.2014.10.011. $\uparrow 4.0 .10,4.0 .10$

[37] O. Röndigs and P. A. Østvær, Motives and modules over motivic cohomology, C. R. Math. Acad. Sci. Paris 342 (2006), no. 10, 751-754, DOI 10.1016/j.crma.2006.03.013. $\uparrow 1$

[38] __ Modules over motivic cohomology, Adv. Math. 219 (2008), no. 2, 689-727, DOI 10.1016/j.aim.2008.05.013. $\uparrow 1,1.3,5.1 .2$

[39] S. Schwede and B. Shipley, Stable model categories are categories of modules, Topology 42 (2003), no. 1, 103-153. $\uparrow 1$

[40] V. Voevodsky, Triangulated categories of motives over a field, Cycles, transfers, and motivic homology theories, 2000, pp. 188-238. $\uparrow 1$

[41] _ Cancellation theorem, Doc. Math. Extra vol.: Andrei A. Suslin sixtieth birthday (2010), 671-685. $\uparrow 4.16$

[42] V. Voevodsky, A. Suslin, and E. Friedlander, Cycles, transfers, and motivic homology theories, Annals of Mathematics Studies, vol. 143, Princeton University Press, Princeton, NJ, 2000. $\uparrow 4$ 